\title{
Changes in cellular ferritin distribution of human brain tissue during autolysis revealed using analytical electron microscopy
}

\author{
Sowmya Sunkara ${ }^{1}$, Snježana Radulović ${ }^{1 *}$, Saška Lipovšek ${ }^{1,2,3,4}$, Christoph Birkl $^{5}$, Stefan Eggenreich ${ }^{6}$, \\ Anna Maria Birkl-Toeglhofer ${ }^{7}$, Maximilian Schinagl ${ }^{1,8,10}$, Daniel Funk ${ }^{1}$, Michael Stöger-Pollach ${ }^{9}$, \\ Johannes Haybaeck ${ }^{7,10}$, Walter Goessler ${ }^{11}$, Stefan Ropele ${ }^{6}$, Gerd Leitinger ${ }^{1}$ \\ ${ }^{1}$ Gottfried Schatz Research Center, Division of Cell Biology, Histology and Embryology, Medical University of Graz, Graz, Austria \\ ${ }^{2}$ Faculty of Medicine, University of Maribor, Maribor, Slovenia \\ ${ }^{3}$ Department of Biology, Faculty of Natural Sciences and Mathematics, University of Maribor, Maribor, Slovenia \\ ${ }^{4}$ Faculty of Chemistry and Chemical Engineering, University of Maribor, Maribor, Slovenia \\ ${ }_{5}^{5}$ University Clinic for Neuroradiology, Medical University of Innsbruck, Innsbruck, Austria \\ ${ }^{6}$ Neuroimaging Research Unit, Department of Neurology, Medical University of Graz, Austria \\ ${ }^{7}$ Institute for Pathology, Neuropathology and Molecular Pathology, Medical University of Innsbruck, Innsbruck, Austria \\ 8 Institute of Chemical Technologies and Analytics, Technische Universität Wien, Vienna, Austria \\ ${ }^{9}$ University Service Centre for Transmission Electron Microscopy (USTEM), Technische Universität Wien, Vienna, Austria \\ ${ }^{10}$ Diagnostic \& Research Center for Molecular BioMedicine, Institute of Pathology, Medical University of Graz, Graz, Austria \\ ${ }^{11}$ Institute for Chemistry, University of Graz, Graz \\ *Correspondence: snjezana.radulovic@medunigraz.at
}

\begin{abstract}
Iron in the human brain is stored in the cores of ferritin proteins. Excess iron has been implicated in neurological disorders, so a careful balance of the iron concentration is thought to be essential for human health. Though it is known that oligodendrocytes contain most of the brain ferritin, followed by other glial cells and neurons, little is known about changes in the cellular ferritin distribution when total iron load changes. Moreover, changes in the number of filled ferritins or in their cellular distribution during autolysis have not been revealed yet. To reveal these changes, we examined brain samples taken from six deceased humans at different post mortem times. To compare samples with different ferritin content, the samples included the frontal gray and frontal white matter, which store a low amount of iron, and the putamen and globus pallidus, which store a high amount of iron. We quantified the number of loaded ferritin cores from iron L - elemental maps obtained with energy filtered transmission electron microscopy and compared this number to the iron concentrations determined using inductively coupled plasma mass spectrometry and quantitative magnetic resonance imaging. The more loaded ferritin cores we found in the tissues, the higher the proportion of ferritins found in neurons instead of glial cells, indicating that total ferritin load scales with the proportion of ferritin that is located in neurons. Second, we found a statistically significant correlation between the post mortem interval and the amount/ concentration of loaded ferritins and found that this concentration is highly dependent on the post mortem interval (PMI): the longer the PMI, the lower the ferritin concentration found in a sample. Third, the longer the post mortem interval, the higher the proportion of ferritins found in neurons instead of glial cells, indicating that ferritin loss during autolysis affects neurons and glial cells at different rates.
\end{abstract}


Key Words

Ferritin, energy-filtered transmission electron microscopy, quantitative magnetic resonance imaging, inductively coupled plasma mass spectrometry, post mortem, human brain, frontal gray matter, frontal white matter, putamen, globus pallidus, autolysis, cellular distribution

\section{Introduction}

Non-heme iron accumulates in the normal ageing brain until the fourth decade of life (Hallgren and Sourander 1958). The accumulation rates and the absolute iron concentration vary strongly across different brain regions: highest concentrations can be found in the basal ganglia including the putamen and the globus pallidus (GP) (Hallgren and Sourander 1958; Krebs et al. 2014). The cause of iron accumulation is still unclear, in particular since much more iron is stored than metabolically relevant.

The total iron concentration in freeze dried tissue samples can be determined most precisely by inductively-coupled plasma mass spectrometry (ICPMS), eg. (Krebs et al. 2014). However, this method is destructive and does not provide information on the various iron-compounds. A non-invasive and thus non-destructive way to measure spatial iron concentrations is quantitative Magnetic Resonance Imaging (qMRI). qMRI allows to mapping the local transverse relaxation rate $\mathrm{R}_{2}{ }^{*}$, which has been validated as a measure for local iron content in brain tissue using mass spectrometry (Langkammer et al. 2010).

It was described previously that ferritin is the main iron storage protein in the brain (Finazzi and Arosio 2014). Ferritin is a protein which is critical for brain functioning, since it is central to iron regulatory mechanisms. Its buffering properties allow it to store and release iron upon cellular demand, which is crucial in prevention of ferroptosis (apoptosis induced by iron overload) (Jiang et al. 2021). High levels of soluble, free iron induce increased reactive oxygen species (ROS) levels (Friedman et al. 2011; Halliwell 2006). Basal levels of iron are essential e.g. in acting as cofactors for enzymatic reactions (Codazzi et al. 2015) and for synaptic plasticity (Muñoz et al. 2011). Labile iron ions can act as free radicals and can lead to neuroferritinopathy followed by neurodegeneration (Friedman et al. 2011).

Ferritin can contain between 500 to 5000 ferric iron atoms in its inner core (Jian et al. 2016; Harrison and Arosio 1996; lancu 2011). The size of ferritin cores in electron micrographs varies depending on various factors such as: ferritin load, contrasting agent, hydration, or the focus of the electron micrograph. Previously published sizes range from $5.3-11 \mathrm{~nm}$ (lancu 2011). While the concentration of total and ferritin bound iron differs between brain areas, the size of ferritin cores remains largely uniform (Friedman et al. 2011).

In regard to size and localization, ferritin takes a unique position amongst the iron - binding proteins. Neither transferrin nor hemoglobin can pass the blood-brain barrier, but ferritin can (Fan et al. 2018), Additionally, transferrin and hemoglobin contain respectively only 2 or 4 iron atoms (Giometto et al. 1993; Marengo-Rowe 2006; Wagner et al. 2003), so they are under the detection threshold for energy - filtered transmission electron microscopy, our method of choice for ferritin detection. On the other hand, hemosiderin is composed of conglomerates of clumped ferritin particles, denatured proteins, and lipids with a size up to 1 - $2 \mu \mathrm{m}$ (Wagner et al. 2003). Accordingly, hemosiderin can be easily distinguished by size from ferritin but is rarely detected in the normal brain. 
In the rat brain, it has been shown that oligodendrocytes store a large amount of iron associated with ferritin (Meguro et al. 2008). In rats, ferritin iron is also contained in pericapillary astrocytes, certain microglial cells and larger neurons (Meguro et al. 2008). While in rat the cellular distribution of ferritins is known, the mechanisms and functions of the different cell types during ferritin storage are not known yet. Post mortem studies have the advantage that the tissue is available for a variety of measurements and procedures, including destructive ones. But immediately after death, autolysis sets off, changing structural components and making identifying them difficult (Sele et al. 2019), reviewed in (Lewis et al. 2019).

In this study we analyzed human brain samples post mortem to answer two fundamental questions: first, we aimed to reveal the role of different cell types in storing ferritin. Second, we aimed to show how iron clustering changes during autolysis. For this, we determined the ferritin concentration in human brain samples using analytical electron microscopy. This procedure allowed us to visualize the chemical elements within a thin tissue section.

Further, we related results from analytical electron microscopy to qMRI and ICPMS measurements of the iron content to obtain complementary insights into effects of sensitivity and autolysis.

\section{Materials and Methods}

\section{Sampling}

The samples used here came from six deceased human subjects who had to undergo routine brain autopsy. The age and post mortem intervals are given in table 1 . To ensure samples were comparable, all patient ages ranged from 61- 86, at which age saturation in iron accumulation has already been reached (Hallgren and Sourander 1958). One brain half was left intact for quantitative magnetic resonance imaging (qMRI) while four brain areas were dissected out of the other brain half and further divided into samples for electron microscopy (EM) and inductively coupled plasma mass spectrometry (ICPMS). The brain regions selected were: the frontal gray matter (FGM), the superficial frontal white matter (FWM), the putamen, and the globus pallidus (GP). As soon as the tissue was cleared for research purposes by the pathologists, samples were dissected out and either immersed in fixative for EM, or placed into a plastic tube, weighed, and frozen for ICPMS.

\section{Electron Microscopy}

Immediately after clearance from the pathologist, 6-10 small samples (maximum dimensions $1 \times 1 \times 1 \mathrm{~mm}^{3}$ ) were cut out of the samples and immersed in chemical fixative containing $2 \%$ formaldehyde, $2 \%$ glutaraldehyde in $0.1 \mathrm{M}$ sodium cacodylate buffer. After fixation the samples were rinsed in the same buffer, post fixed in $1 \%$ osmium tetroxide solution in the same buffer, dehydrated in a graded series of alcohols, immersed in propylene oxide, and embedded in TAAB embedding resin (TAAB, Aldermaston, UK). After curing for 3 days at $60{ }^{\circ} \mathrm{C}$, thin sections were cut using a Leica UC6 or a Leica UC7 ultramicrotome at a thickness of either 60 or $70 \mathrm{~nm}$. The sections were contrasted using platinum blue and lead citrate solutions.

Electron microscopy was performed on one section from a randomly selected block, with a Thermo Fisher Tecnai 20 electron microscope operated at 200 kV. An Ametek Gatan Quantum GIF energy filter with 
Ametek Gatan Ultrascan 1000 XP camera was used for energy- filtered transmission electron microscopy (EFTEM), and a bottom-mounted Ametek Gatan Ultrascan 1000 camera for bright field imaging.

For elemental mapping, three windows were made at $80000 \times$ magnification, a binning of 2 , a slot width of $40 \mathrm{eV}$, and an exposure time of $30 \mathrm{~s}$. The energy losses were: $718 \mathrm{eV}$ for the iron L- post edge window, and 633 and $673 \mathrm{eV}$ for the two pre-edge windows. Drift correction was done manually, and both an elemental map and a ratio map were calculated using respectively the three window method and the twowindow method.

An unbiased sampling protocol described earlier ( Wernitznig et al. 2019) allowed us to automatically move each sample to 60 locations determined using a random number generator. The elemental maps were accompanied by overview micrographs taken from the same locations using the bright field mode at 3500 $\mathrm{x}$ magnification.

\section{Quantitating the number of ferritins and assessing cell types}

Four independent researchers (S.S., S.R., S.L, and G.L.) each counted the number of iron-loaded ferritins and assessed the cell types. Ferritins were only counted if they were clearly visible on both the jump ratio and the elemental maps, and if at least three of the four researchers agreed on their number (otherwise images were analyzed again and all researchers counted ferritins simultaneously). Means of the four researchers' numbers were made for counts of 20 or more. Cell types were identified on the bright field images and only noted if three of the four researchers agreed. For cell identification the criteria described by (Nahirney and Tremblay 2021), and the Boston University web atlas https://www.bu.edu/agingbrain/ (download 2022-01-14) were used.

\section{Quantitative Magnetic Resonance Imaging}

MR imaging of the extracted brain hemisphere was performed using a 3.0 Tesla scanner (MAGNETOM PRISMA, Siemens Healthineers, Erlangen, Germany). A phased array head coil with 20 elements was used. For acquiring $\mathrm{R}_{2}$ * relaxation data, a two-dimensional radiofrequency-spoiled multi-echo gradient-echo sequence with 6 equally spaced echoes was applied (repetition time $=1150 \mathrm{~ms}$, first echo time $=4.92 \mathrm{~ms}$, echo spacing $=5.42 \mathrm{~ms}$, flip angle $=15^{\circ}$; field of view $=256 \times 256 \mathrm{~mm}^{2} ;$ in-plane resolution $=0.75 \times 0.75$ $\mathrm{mm}^{2}$; 20 slices; 4-mm-thick sections; and acquisition time of 7 minutes 35 seconds). Generalized autocalibrating partially parallel acquisition was performed with an acceleration factor of 2 .

To reduce phase dispersion effects and to increase B0 homogeneity, second-order shimming was applied. All images were acquired in axial orientation and angulated parallel to the most inferior parts of the occipital and frontal lobes. The single section used for $\mathrm{R}_{2}{ }^{*}$ mapping was angulated parallel to the axial sections of the three-dimensional gradient-echo sequence and centered in the middle of the basal ganglia. R2* maps were calculated with the relaxometry toolbox ( (ESMRMB 2016, 33rd Annual Scientific Meeting, Vienna, AT, September 29 - October 1: ePoster / Paper Poster / Clinical Review Poster / Software Exhibits 2016). 


\section{Inductively Coupled Plasma Mass spectrometry}

All solutions were prepared with ultrapure water (18.2 $\mathrm{M} \Omega^{*} \mathrm{~cm}$, Merck Millipore, Bedford, USA). Nitric acid (>65\% p.a.) was further purified via sub-boiling. For quantification single elements standards (ROTI ${ }^{\circledR}$ Star, Carl Roth GmbH + Co. KG, Karlsruhe, Germany) were used.

Brain tissue was freeze-dried to constant mass with a Gamma 1-16 LSC freeze-dryer (Martin Christ GmbH, Osterode am Harz, Germany) and the wet/dry mass ratio determined. Freeze-dried samples were weighed to $0.1 \mathrm{mg}$ into the $12 \mathrm{~mL}$ quartz vessels of a microwave-heated autoclave, UltraCLAVE IV (EMLS, Leutkirch, Germany). After addition of $3 \mathrm{~mL}$ subboiled nitric acid and $2 \mathrm{~mL}$ of water the quartz vessels were placed in the 40 positions rack of the autoclave and the autoclave pressurized with Argon to a pressure of $4 * 10^{6} \mathrm{~Pa}$. The following microwave heating program was applied: from room temperature to $80^{\circ} \mathrm{C}$ in 5 minutes, from $80^{\circ} \mathrm{C}$ to $150^{\circ} \mathrm{C}$ in 15 minutes, from $150^{\circ} \mathrm{C}$ to $250^{\circ} \mathrm{C}$ in 20 minutes, $250^{\circ} \mathrm{C}$ for 30 minutes. After cooling to $80^{\circ} \mathrm{C}$, pressure was released and the samples were transferred to $50 \mathrm{~mL}$ polypropylene tubes (CELLSTAR ${ }^{\circledR}$, blue screw cap, Greiner Bio-One $\mathrm{GmbH}$, Kremsmuenster, Austria) and filled to the mark.

For the quantification of magnesium ( $\mathrm{Mg})$, calcium ( $\mathrm{Ca})$, iron ( $\mathrm{Fe})$, copper $(\mathrm{Cu})$ and zinc $(\mathrm{Zn})$ calibration curves were prepared from 0.0100 to 10.0 ( $\mathrm{Mg}, \mathrm{Ca}, \mathrm{Fe}$ ) and from 0.00100 to $1.00 \mathrm{mg} / \mathrm{L}$ for $\mathrm{Cu}$ and $\mathrm{Zn}$. Germanium (Ge) at a concentration of $200 \mu \mathrm{g} / \mathrm{L}$ was added as internal standard online before the nebulizer. The samples were pumped through a peristaltic pump tubing with an i.d. of $1.05 \mathrm{~mm}$ and the internal standard with a tubing of $0.19 \mathrm{~mm}$. The elements were quantified with an inductively coupled plasma mass spectrometer (ICPMS, Agilent 7700x, Agilent Technologies, Waldbronn, Germany) at the following mass-to-charge ratios: $\mathrm{Ca} \mathrm{m} / \mathrm{z}=43, \mathrm{Mg} \mathrm{m} / \mathrm{z}=24, \mathrm{Fe} \mathrm{m} / \mathrm{z}=56, \mathrm{Cu} \mathrm{m} / \mathrm{z}=65$ and $\mathrm{Zn} \mathrm{m} / \mathrm{z}=66$. All isotopes were measured in the collision mode using He at a flow rate of $5.0 \mathrm{ml} / \mathrm{min}$. The determined concentrations were calculated to fresh tissue.

Accuracy of the determined concentrations was confirmed with the certified material bovine muscle (RM 8414; NIST; now BOVM-1 NRCC).

\section{Statistical Analysis and Graphs}

Statistics were done using GraphPadPrism 9.2.0. To avoid bias in the correlation of $\mathrm{R}_{2}{ }^{*}$ and iron content or ferritin count, the white matter samples were omitted from those analyses that correlated data obtained with qMRI with data obtained with ICPMS or EM. This was because white matter contains a high number of myelin sheaths and the diamagnetic effect of myelin sheaths had previously been shown to counteract the paramagnetic effect of iron on $\mathrm{R}_{2}{ }^{*}$ (Birkl et al. 2015; Birkl et al. 2018).

\section{Results}

\section{EFTEM elemental mapping allows visualising ferritins cores in human post mortem brain tissue}

In order to visualize ferritin cores in electron micrographs, we made iron L- elemental maps and ratio maps of brain samples for four different brain areas in each of six different deceased. The maps consistently exhibited bright spots several nanometers in diameter (Fig. 1A-D). The size of the spots corresponded to the published sizes of ferritin cores (lancu 2011), indicating that filled ferritin cores could be visualized. 


\section{Ferritin cores were clustered in distinct cells}

We next counted the number of ferritin cores in samples taken from the frontal cortex, the frontal white matter, the putamen, and the globus pallidus of each patient. The counts were made within 60 micrographs of $556 \times 556 \mathrm{~nm}^{2}$ dimensions and showed that the ferritins were not evenly distributed but clustered in certain micrographs (Fig. 2). At closer examination it became apparent that certain cells accumulated ferritins, whereas other, often neighboring cells, did not accumulate ferritins (Fig. 1D). None of the ferritins appeared to be extracellular and we were able to assess the cell type (neuron, oligodendrocyte, or other or unidentified glial cell) in 2,195 (62\%) of the 3,537 ferritin cores that we had identified in five out of six samples. 471 additional ferritin cores were identified in the sixth sample with a post mortem interval of $24 \mathrm{~h}$, but in this sample autolysis had progressed considerably, preventing us from faithfully identifying cell types. Accordingly, this sample was excluded from cell type identification experiments.

\section{Ferritins accumulate in the putamen and globus pallidus}

We next calculated the number of ferritins per $\mu \mathrm{m}^{3}$ from the micrographs' 3D dimensions. Plotting this ferritin concentration in each brain region for each deceased person revealed that either the putamen, the globus pallidus, or both, had a significantly higher concentration of ferritins than either the frontal gray matter or the frontal white matter (Fig. 3). Significant differences in ferritin concentrations were also detected between the frontal gray and frontal white matter, but only in one out of six patients. Further, between the putamen and globus pallidus (4 out of 6 patients) significant differences were found (Fig. 3). The putamen had a significantly higher ferritin concentration than the globus pallidus in one out of six patients, and the globus pallidus had a significantly higher concentration compared to the putamen in three out of six patients (Fig. 3).

\section{qMRI and ICPMS data support the EFTEM data}

The ferritin concentrations counted from elemental maps were compared with the mean iron concentrations. The latter were determined by either mass spectrometry in adjacent tissue samples, or by the proton transverse relaxation rate $\left(R_{2}{ }^{*}\right)$ - a qMRI measure proportional to the iron concentration measured in the contralateral hemisphere (Fig. 4). Each of the three methodological approaches provided higher mean values in the putamen and globus pallidus when compared to the frontal gray and frontal white matter (Fig. 4). Significant differences were found in the iron concentrations between putamen and frontal gray matter using mass spectrometry (Fig. 4B). Quantitative magnetic resonance imaging revealed a significant $\mathrm{R} 2 *$ increase in frontal white and frontal gray matter compared to the putamen.

To compare the three different methodological approaches, the mean values of the measured brain regions from all six deceased persons were correlated with each other. There was a high correlation between $\mathrm{qMRI}$ and ICPMS results, each measuring the iron concentration. The higher the $\mathrm{R}_{2}{ }^{*}$ value, the higher the iron concentration (Fig. 5Aa, slope differs significantly from 0 ). However, we found no correlation between qMRI data and the mean ferritin count obtained using EFTEM (Fig. 5B), nor between the mean ferritin count and the iron concentration (Fig. 5C). 


\section{Cell type identification shows that most ferritins are contained in glial cells, especially oligodendrocytes}

The ferritin distribution in different cell types showed, that most of the 2195 ferritin cores which could be assigned to specific cell types were found within glial cells. Oligodendrocytes represented the major cell type within this fraction (56 \%. Fig. 6). $19 \%$ were found in neurons (Fig. 6).

\section{The percentage of ferritin cores found in neurons increased with increasing ferritin count}

To evaluate whether cell specific accumulation of ferritin is dependent on the total ferritin amount, we plotted the percentage of ferritin found in neurons against the total ferritin count in the gray or white matter in each sample. This analysis showed that the percentage of ferritins stored in neurons increased with increasing ferritin count. This result indicates that, although the majority of ferritins was situated in glial cells, ferritin storage occurred disproportionately more in neurons compared to other cell types (Fig. 7).

\section{The post mortem interval had an influence on the mean ferritin count and on the cellular distribution of ferritins}

We next tested whether putative autolysis during the post mortem interval had an influence on the iron concentration, the storage of iron in ferritins, or the cellular distribution of the ferritins.

Our analysis showed that the mean ferritin count was inversely correlated to the post mortem interval (Fig. 8A). In contrast, neither the $\mathrm{R}_{2}{ }^{*}$ value nor the MS determined iron concentration correlated with the post mortem interval (Fig. 8B, C).

Further the percentage of ferritins found using EFTEM in neurons significantly decreased with increasing post-mortem interval (Fig. 8D). This indicates that the longer the post mortem time, the lower the proportion of ferritins found in neurons, and the higher the proportion of ferritins found in glial cells.

\section{Discussion}

In this study we used analytical electron microscopy to determine the concentration of iron-loaded ferritin cores in the human brain. Thin sections taken from four brain regions of six deceased persons were examined. The persons had not suffered from a known neurological disorder and the samples were taken during routine autopsies. We aimed to validate the assessment of local iron-loaded ferritin concentrations with data on the $\mathrm{R}_{2}$ * relaxation rate obtained using $\mathrm{qMRI}$ (a marker for iron in brain tissue), and with the iron concentrations in neighboring tissue samples obtained using MS.

Due to the electron density of the ferritins iron cores, they are directly visible in classical electron micrographs (reviewed in (lancu 2011)), but they can become obscured by the contrasting agent in routine contrasted sections (Iancu 2011). Accordingly, we chose an energy filter to create iron maps for trustworthy ferritin core identification. The size (lancu 2011) and detectability of these clusters indicates that they correspond to the iron-loaded cores of ferritins. After identifying the ferritin cores, we compared the elemental maps with a classical electron micrograph of the same area for cell identification and ferritin 
localization. We were able to demonstrate that the putamen and the GP store more ferritin iron clusters than the FGM and the FWM, confirming a trend also seen in data obtained with qMRI and MS.

It has to be borne in mind that the EFTEM method is based on visualizing iron clusters using analytical electron microscopy. Ferritins are known to vary in their iron load (Jian et al. 2016; Harrison and Arosio 1996; lancu 2011) and those ferritin proteins that contain a low amount of iron remain undetectable using EFTEM. Instead of using a fixed detection threshold, this study relied on four independent researchers agreeing on the ferritin count from both iron L-elemental maps and jump ratios.

Although we have no means of counting empty ferritin molecules or ferritin molecules with a low iron load, we nevertheless were able to demonstrate that the putamen and globus pallidus had a higher mean concentration of detectable (iron-loaded) ferritins than the frontal gray and white matter. That the basal ganglia are storage sites for iron has long been known (Hallgren and Sourander 1958) and has since been confirmed in several different studies that used a variety of different methods (e.g. Maeda et al., 1997, Morris 1992, Krebs et al., 2014, Ramos 2014). To validate our findings, we measured the iron concentration in adjacent pieces of tissue taken from the same patients with ICPMS, and measured the proton relaxation rate $R_{2}{ }^{*}$, using $q M R I$ in the contralateral region of the same patients. Both methods were in good agreement and confirmed that the basal ganglia have both higher iron concentrations and a higher proton relaxation rate than the frontal cortex and gray matter. This is also in line with an earlier study of post mortem human brain tissue, in which qMRI was combined with MS to determine the relative iron concentrations within the tissue (Langkammer, 2010).

Moreover, we were able to determine the cell type (neuron, oligodendrocyte or other glial cell) that contained most (62\%) of the ferritins we had detected in five out of the six patients. We found that most of the iron in the human brain is stored in oligodendrocytes, but iron is also found in other or unidentifiable glial cells and neurons. Earlier studies support our results using a variety of different techniques (Meguro et al. 2008; Connor et al. 1990; Quintana et al. 2006; Quintana et al. 2004). Interestingly, we found that the probability of the ferritins being stored in neurons rather than glial cells correlates positively with total ferritin concentration in each patient. To our knowledge, this is the first study that shows that total ferritin load scales with the proportion of ferritin that is located in neurons.

When plotting the mean proton relaxation rate against the mean iron concentration from the gray matter of each patient, we found a high correlation between proton relaxation rate and iron level, confirming an earlier, comparative study of both methods (Krebs et al., 2014). In contrast, the mean ferritin concentration of all the brain areas did not correlate with the mean iron concentration of each patient, indicating that the number of loaded ferritins found in each patient was not directly dependent on the total iron concentration. We thus looked for a factor that has an influence on the concentration of loaded ferritins and found that this concentration is highly dependent on the post mortem interval (PMI): the longer the PMI, the fewer ferritins are found in a sample. This indicates that autolysis during the post mortem time leads to either unloading or decay of ferritins. In contrast, neither the proton relaxation rate nor the total iron content were affected by the post mortem interval. This signifies that the iron is not lost during that interval, indicating that the proton relaxation rate represents the iron load of a specific brain area independent of the post mortem interval. Further studies will be necessary to detect possible changes in oxidation state during the unloading or decay of ferritins. 
When looking into which cell types are involved in iron loss during the post mortem interval, we again found that the loss occurs at a higher rate in neurons than in glial cells. Neurons were shown to have a different ferritin isoform distribution compared with glial cells (Connor et al. 1994; Nash et al. 2019). Moreover, when ferritin $\mathrm{H}$ (a subtype containing a high number of heavy chains) is selectively deleted in neurons, the protective effect of melatonin against ferroptosis is largely reduced (Rui et al. 2021) indicating that ferroptosis appears to be mainly driven by neurons. We thus can only speculate as to the cellular mechanisms that govern this loss of loaded ferritins during the post mortem interval. But we have strong evidence that neurons are involved in it at a higher rate than glial cells.

In summary, we found high involvement of neurons in the storage of ferritins. Moreover, iron loaded ferritins are lost post mortem, in a yet unknown process, and loss of ferritins occurs at a higher rate in neurons than in glial cells.

Abbreviations:

EFTEM - energy - filtered transmission electron microscopy

FGM - frontal gray matter

FWM - frontal white matter

GP - globus pallidus

ICPMS - inductively - coupled plasma mass spectrometry

qMRI - quantitative magnetic resonance imaging

\section{Acknowledgements:}

We are grateful to Dr. Sigurd Lax, for advice and for contributing material, Dr Peter Simmons (Newcastle University, UK), for proof reading and language corrections, Mariella Sele, for processing and evaluating some of the samples, Elisabeth Bock, Elisabeth Pritz, Luca Schmid, Susanne Sumerauer and Nina Schlögl for expert technical assistance.

The study design was approved by the Ethics Committee of the Medical University of Graz, votum number 28-549 ex 15/16.

Funded by the Austrian Science Foundation, grant P 29370.

\section{Publication bibliography}

Birkl, Christoph; Carassiti, Daniele; Hussain, Fariha; Langkammer, Christian; Enzinger, Christian; Fazekas, Franz et al. (2018): Assessment of ferritin content in multiple sclerosis brains using temperature-induced $\mathrm{R}^{*} 2$ changes. In Magnetic resonance in medicine 79 (3), pp. 1609-1615. DOI: 10.1002/mrm.26780.

Birkl, Christoph; Langkammer, Christian; Krenn, Heinz; Goessler, Walter; Ernst, Christina; Haybaeck, Johannes et al. (2015): Iron mapping using the temperature dependency of the magnetic susceptibility. In Magnetic resonance in medicine 73 (3), pp. 12821288. DOI: $10.1002 / \mathrm{mrm} .25236$.

Codazzi, Franca; Pelizzoni, Ilaria; Zacchetti, Daniele; Grohovaz, Fabio (2015): Iron entry in neurons and astrocytes: a link with synaptic activity. In Frontiers in molecular neuroscience 8, p. 18. DOI: 10.3389/fnmol.2015.00018.

Connor, J. R.; Boeshore, K. L.; Benkovic, S. A.; Menzies, S. L. (1994): Isoforms of ferritin have a specific cellular distribution in the brain. In Journal of neuroscience research 37 (4), pp. 461-465. DOI: 10.1002/jnr.490370405. 
Connor, J. R.; Menzies, S. L.; St Martin, S. M.; Mufson, E. J. (1990): Cellular distribution of transferrin, ferritin, and iron in normal and aged human brains. In Journal of neuroscience research 27 (4), pp. 595-611. DOI: 10.1002/jnr.490270421.

ESMRMB 2016, 33rd Annual Scientific Meeting, Vienna, AT, September 29 - October 1: ePoster / Paper Poster / Clinical Review Poster / Software Exhibits (2016). In Magma (New York, N.Y.) 29 Suppl 1, pp. 401-475.

Fan, Kelong; Jia, Xiaohua; Zhou, Meng; Wang, Kun; Conde, João; He, Jiuyang et al. (2018): Ferritin Nanocarrier Traverses the Blood Brain Barrier and Kills Glioma. In ACS nano 12 (5), pp. 4105-4115. DOI: 10.1021/acsnano.7b06969.

Finazzi, Dario; Arosio, Paolo (2014): Biology of ferritin in mammals: an update on iron storage, oxidative damage and neurodegeneration. In Archives of toxicology 88 (10), pp. 1787-1802. DOI: 10.1007/s00204-014-1329-0.

Friedman, Andrzej; Arosio, Paolo; Finazzi, Dario; Koziorowski, Dariusz; Galazka-Friedman, Jolanta (2011): Ferritin as an important player in neurodegeneration. In Parkinsonism \& related disorders 17 (6), pp. 423-430. DOI: 10.1016/j.parkreldis.2011.03.016.

Giometto, Bruno; Gallo, Paolo; Tavolato, Bruno (1993): Transferrin Receptors in the Central Nervous System. In P. Michael Conn (Ed.): Methods in neurosciences, vol. 11: Elsevier (Methods in Neurosciences), pp. 122-134.

Hallgren, B.; Sourander, P. (1958): The effect of age on the non-haemin iron in the human brain. In Journal of neurochemistry 3 (1), pp. 41-51. DOI: 10.1111/j.1471-4159.1958.tb12607.x.

Halliwell, Barry (2006): Oxidative stress and neurodegeneration: where are we now? In Journal of neurochemistry 97 (6), pp. 16341658. DOI: 10.1111/j.1471-4159.2006.03907.x.

Harrison, Pauline M.; Arosio, Paolo (1996): The ferritins: molecular properties, iron storage function and cellular regulation. In Biochimica et Biophysica Acta (BBA) - Bioenergetics 1275 (3), pp. 161-203. DOI: 10.1016/0005-2728(96)00022-9.

lancu, Theodore C. (2011): Ultrastructural aspects of iron storage, transport and metabolism. In Journal of neural transmission (Vienna, Austria : 1996) 118 (3), pp. 329-335. DOI: 10.1007/s00702-011-0588-7.

Jian, Nan; Dowle, Miriam; Horniblow, Richard D.; Tselepis, Chris; Palmer, Richard E. (2016): Morphology of the ferritin iron core by aberration corrected scanning transmission electron microscopy. In Nanotechnology 27 (46), 46LT02. DOI: 10.1088/09574484/27/46/46LT02.

Jiang, Xuejun; Stockwell, Brent R.; Conrad, Marcus (2021): Ferroptosis: mechanisms, biology and role in disease. In Nature reviews. Molecular cell biology 22 (4), pp. 266-282. DOI: 10.1038/s41580-020-00324-8.

JoVE Video Dataset.

Krebs, Nikolaus; Langkammer, Christian; Goessler, Walter; Ropele, Stefan; Fazekas, Franz; Yen, Kathrin; Scheurer, Eva (2014): Assessment of trace elements in human brain using inductively coupled plasma mass spectrometry. In Journal of trace elements in medicine and biology : organ of the Society for Minerals and Trace Elements (GMS) 28 (1), pp. 1-7. DOI: 10.1016/j.jtemb.2013.09.006.

Langkammer, Christian; Krebs, Nikolaus; Goessler, Walter; Scheurer, Eva; Ebner, Franz; Yen, Kathrin et al. (2010): Quantitative MR imaging of brain iron: a postmortem validation study. In Radiology 257 (2), pp. 455-462. DOI: 10.1148/radiol.10100495.

Langkammer, Christian; Ropele, Stefan; Pirpamer, Lukas; Fazekas, Franz; Schmidt, Reinhold (2014): MRI for iron mapping in Alzheimer's disease. In Neuro-degenerative diseases 13 (2-3), pp. 189-191. DOI: 10.1159/000353756.

Lewis, Amanda J.; Genoud, Christel; Pont, Mélissa; van de Berg, Wilma Dj; Frank, Stephan; Stahlberg, Henning et al. (2019): Imaging of post-mortem human brain tissue using electron and X-ray microscopy. In Current opinion in structural biology 58, pp. 138-148. DOI: 10.1016/j.sbi.2019.06.003.

Marengo-Rowe, Alain J. (2006): Structure-function relations of human hemoglobins. In Proceedings (Baylor University. Medical Center) 19 (3), pp. 239-245. DOI: 10.1080/08998280.2006.11928171.

Meguro, Reiko; Asano, Yoshiya; Odagiri, Saori; Li, Chengtai; Shoumura, Kazuhiko (2008): Cellular and subcellular localizations of nonheme ferric and ferrous iron in the rat brain: a light and electron microscopic study by the perfusion-Perls and -Turnbull methods. In Archives of histology and cytology 71 (4), pp. 205-222. DOI: 10.1679/aohc.71.205.

Muñoz, Pablo; Humeres, Alexis; Elgueta, Claudio; Kirkwood, Alfredo; Hidalgo, Cecilia; Núñez, Marco T. (2011): Iron mediates Nmethyl-D-aspartate receptor-dependent stimulation of calcium-induced pathways and hippocampal synaptic plasticity. In The Journal of biological chemistry 286 (15), pp. 13382-13392. DOI: 10.1074/jbc.M110.213785. 
Nahirney, Patrick C.; Tremblay, Marie-Eve (2021): Brain Ultrastructure: Putting the Pieces Together. In Frontiers in cell and developmental biology 9, p. 629503. DOI: 10.3389/fcell.2021.629503.

Nash, Bradley; Tarn, Kevin; Irollo, Elena; Luchetta, Jared; Festa, Lindsay; Halcrow, Peter et al. (2019): Morphine-Induced Modulation of Endolysosomal Iron Mediates Upregulation of Ferritin Heavy Chain in Cortical Neurons. In eNeuro 6 (4). DOI: 10.1523/ENEURO.0237-19.2019.

Quintana, C.; Bellefqih, S.; Laval, J. Y.; Guerquin-Kern, J. L.; Wu, T. D.; Avila, J. et al. (2006): Study of the localization of iron, ferritin, and hemosiderin in Alzheimer's disease hippocampus by analytical microscopy at the subcellular level. In Journal of structural biology 153 (1), pp. 42-54. DOI: 10.1016/j.jsb.2005.11.001.

Quintana, C.; Cowley, J. M.; Marhic, C. (2004): Electron nanodiffraction and high-resolution electron microscopy studies of the structure and composition of physiological and pathological ferritin. In Journal of structural biology 147 (2), pp. 166-178. DOI: 10.1016/j.jsb.2004.03.001.

Rui, Tongyu; Wang, Haochen; Li, Qianqian; Cheng, Ying; Gao, Yuan; Fang, Xuexian et al. (2021): Deletion of ferritin H in neurons counteracts the protective effect of melatonin against traumatic brain injury-induced ferroptosis. In Journal of pineal research 70 (2), e12704. DOI: 10.1111/jpi.12704.

Sele, Mariella; Wernitznig, Stefan; Lipovšek, Saška; Radulović, Snježana; Haybaeck, Johannes; Birkl-Toeglhofer, Anna Maria et al. (2019): Optimization of ultrastructural preservation of human brain for transmission electron microscopy after long post-mortem intervals. In Acta neuropathologica communications 7 (1), p. 144. DOI: 10.1186/s40478-019-0794-3.

Wagner, Kenneth R.; Sharp, Frank R.; Ardizzone, Timothy D.; Lu, Aigang; Clark, Joseph F. (2003): Heme and iron metabolism: role in cerebral hemorrhage. In Journal of cerebral blood flow and metabolism : official journal of the International Society of Cerebral Blood Flow and Metabolism 23 (6), pp. 629-652. DOI: 10.1097/01.WCB.0000073905.87928.6D.

Wernitznig, Stefan; Reichmann, Florian; Sele, Mariella; Birkl, Christoph; Haybäck, Johannes; Kleinegger, Florian et al. (2019): An Unbiased Approach of Sampling TEM Sections in Neuroscience. In Journal of visualized experiments : JoVE (146). DOI: $10.3791 / 58745$.

\section{Table and figures}

\section{Table 1. Patient information}

Figure 1. A. Example TEM-overview image of a human brain sample used for EFTEM elemental mapping. B. Inverted post-edge image of region of interest chosen from overview image. C. corresponding iron (L) ratio map of the region of interest. D. corresponding iron (L) elemental map of the region of interest. Red arrows point to iron within ferritin cores.

Figure 2. The ferritin concentration in different brain regions varies between patients 1-6. A. FGM. B FWM. C. putamen. D. GP. Data are shown as bar graphs with whiskers representing the mean +/- SEM. Each dot represents the ferritin concentration measured in one iron elemental map, $(n=60$ for each patient in each brain region).

Figure 3. The ferritin concentration is often, but not always, higher in the basal ganglia than in the frontal cortex or frontal white matter. Graphs show the ferritin concentration in the FGM, FWM, putamen, and GP in patient $1(A)$, patient $2(B)$, patient $3(C)$, patient $4(D)$, patient $5(E)$, and patient $6(F)$. Data are shown as bar graphs with whiskers representing the mean +/-SEM. Each dot represents the ferritin concentration calculated from counts in one iron elemental map, respectively $(n=60$ for each brain region and each patient). One-way ANOVA with a Turkey's multiple comparisons test was used for statistical analysis with $* \mathrm{p}<0.05 ; * * \mathrm{p}<0.01 ; * * * * \mathrm{p}<0.0001$. 
Figure 4. The differences in ferritin concentrations between brain areas that had been determined using EFTEM follow the same trends as mass spectrometry (MS) and quantitative magnetic resonance imaging (qMRI) data in adjacent samples (MS) or samples from the other brain half (qMRI). A. Ferritin concentration in the FGM, FWM, putamen, and GP measured by EFTEM. B. Iron concentration in the FGM, FWM, putamen, and GP measured by ICPMS. C. Proton transverse relaxation rate $\left(R_{2}{ }^{*}\right)$ in the FGM, FWM, putamen, and GP measured by qMRI.Data are shown as bar graphs with whiskers representing the mean +/- SEM. Each dot represents the mean ferritin concentration measured in one patient, respectively $(n=$ 6). One-way ANOVA with a Turkey's multiple comparisons test was used for statistical analysis with * $\mathrm{p}<$ $0.05 ; * * * p<0.001 ; * * * * p<0.0001$.

Figure 5. The mean proton transverse relaxation rate of each patient correlates significantly with the mean iron concentration. B In contrast, the mean concentration of ferritin particles does not correlate with iron concentration nor with the proton relaxation rate. A. Proton transverse relaxation rate (qMRI) plotted over the iron concentration (ICPMS) for each. B. Mean ferritin concentration (EFTEM) plotted over the proton transverse relaxation rate (qMRI). C. Mean ferritin concentration (EFTEM) plotted over the iron concentration (ICPMS). Linear correlations were calculated and the correlation coefficients $r^{2}$ were, 0.8537 for $A, 0.0452$ for $B$, and 0.0043 for $C$. Plots are based on mean values of the FGM, the putamen, and the GP for $A$ and $B$, and for the FGM, the FWM, the putamen, and the GP for C.

Figure 6. Ferritin is predominately stored in glial cells (81 \%) and especially in oligodendrocytes (56 \%); 19 $\%$ is stored in neurons. The graph shows those ferritin particles in the FGM, the putamen, and the GP of patients 1-5 whose cell type could be determined.

Figure 7. The probability of ferritins being found in neurons rises with the total ferritin amount (x-axis). A. Mean values of the \% of ferritins found in neurons in the FGM, the putamen, and the GP for patients 1-5. Correlation coefficient $\left(r^{2}=0.7322\right)$.

Figure 8. Ferritin particles disintegrate and their probability of being found in neurons is reduced over time post-mortem ( $x$-axis of each graph). A. Mean values of the ferritin concentration (EFTEM) in all the brain regions, $r^{2}=0.6421$. B. Mean values of the $\%$ of ferritin concentration in neurons (EFTEM). $r^{2}=0.8338$. C. Mean values of the proton transverse relaxation rate ( $q M R I)$ in the FGM, the putamen, and the GP, $r^{2}=$ 0.1414 . D. Mean values of the iron concentration (MS) in all the brain regions. $r^{2}=0.1215$ ). 
Table 1

\begin{tabular}{|c|c|c|}
\hline Patient code & Age [years] & PMI [h] \\
\hline 1 & 73 & $06: 30$ \\
2 & 72 & 9 \\
3 & 61 & 16 \\
4 & 69 & 18 \\
5 & 86 & 20 \\
6 & 81 & 24 \\
\hline
\end{tabular}


Figure 1

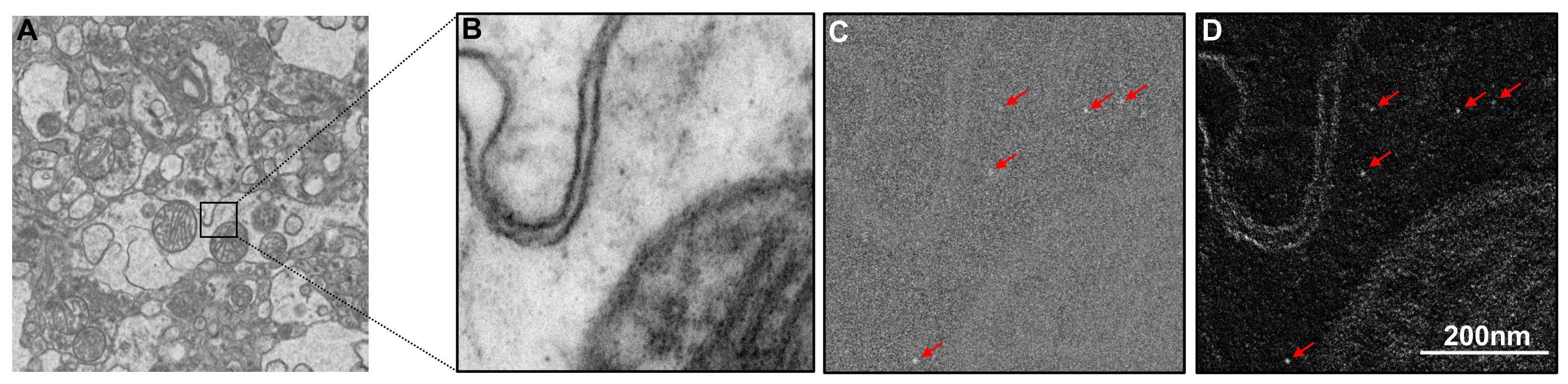




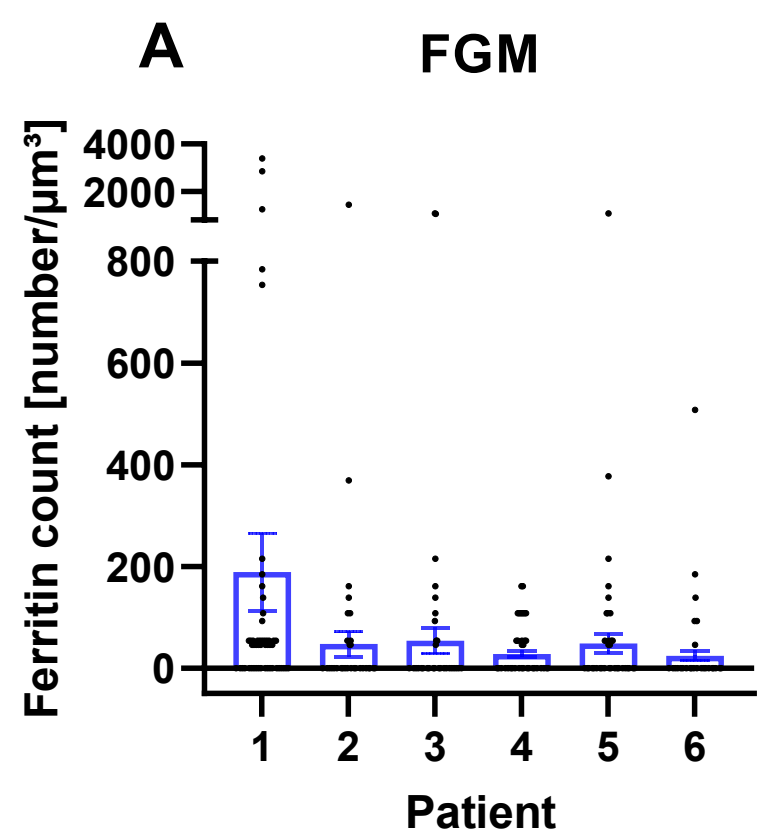

\section{Putamen}
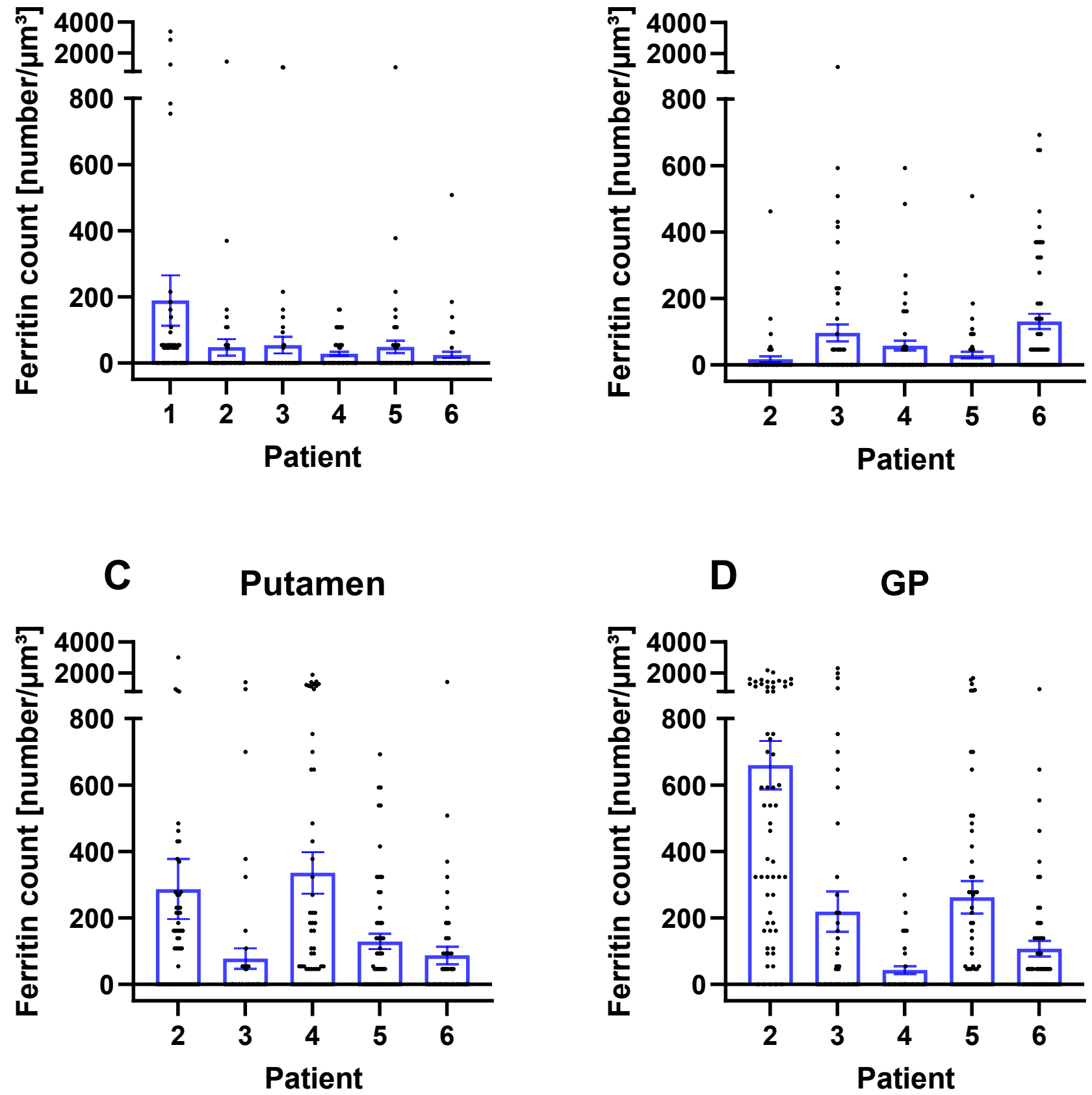


\section{Figure 3}

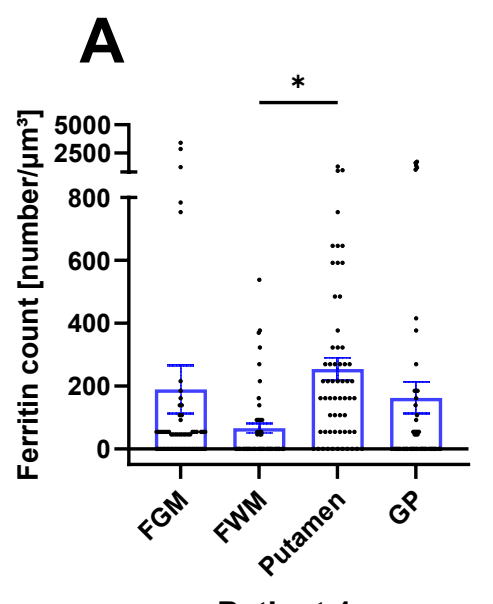

Patient 1

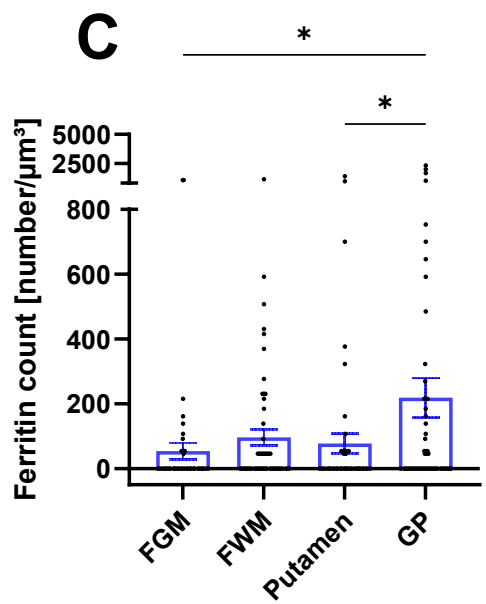

Patient 3

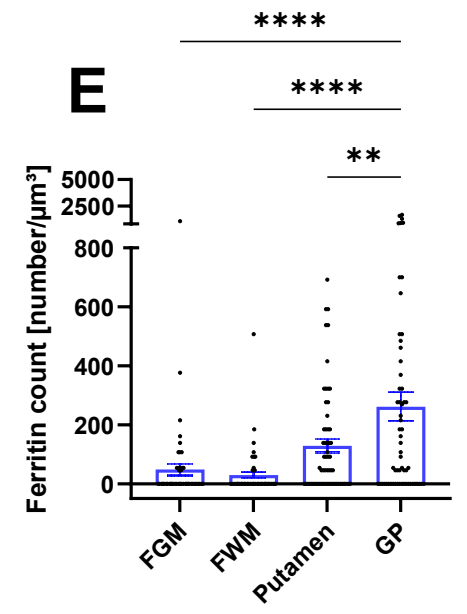

Patient 5

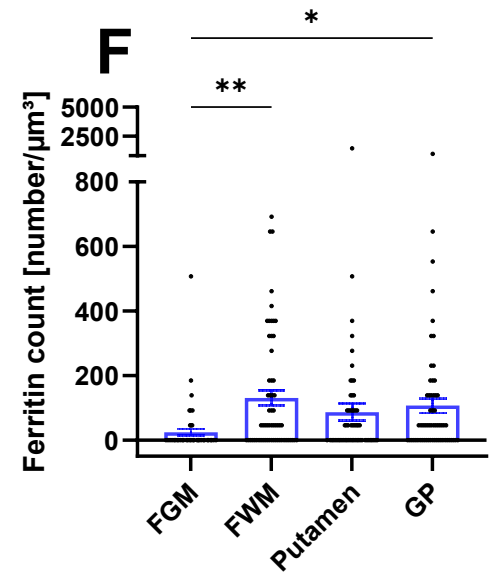

Patient 6 
Figure 4
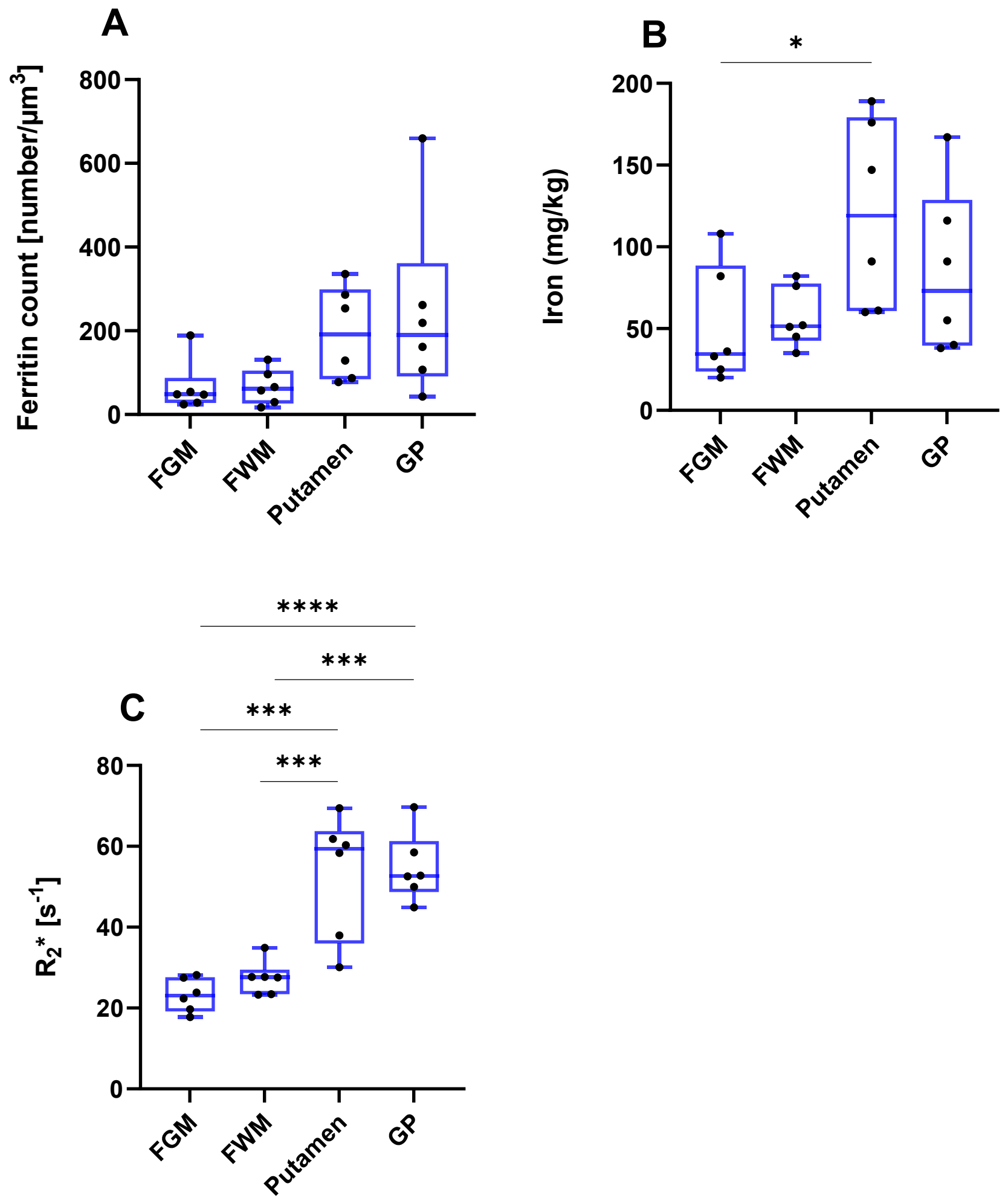

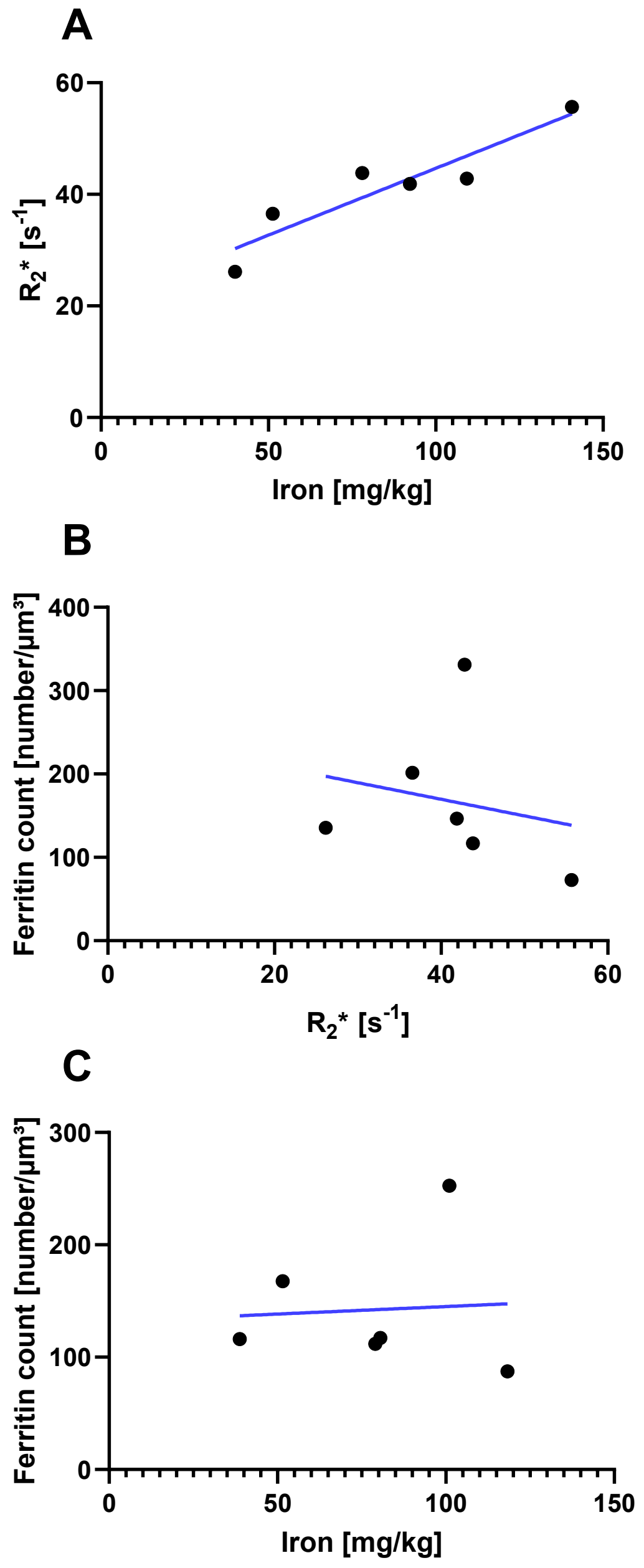


\section{Figure 6}

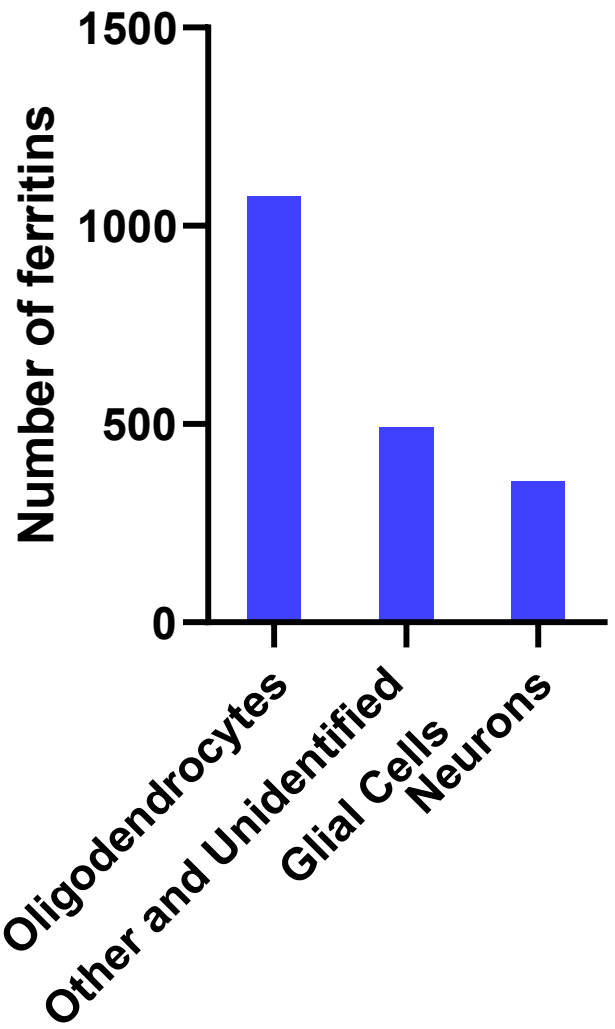


Figure 7

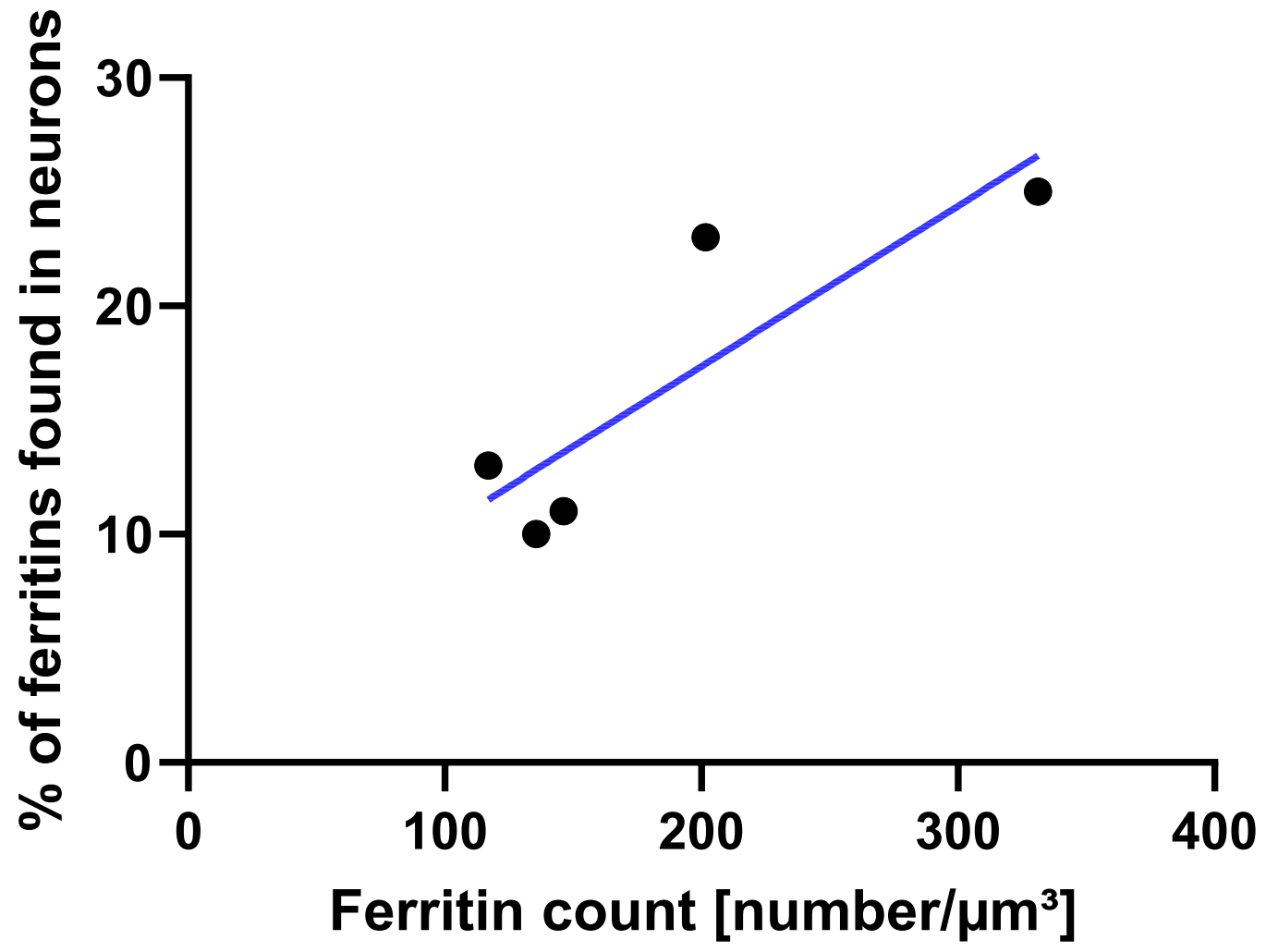


Figure 8

A

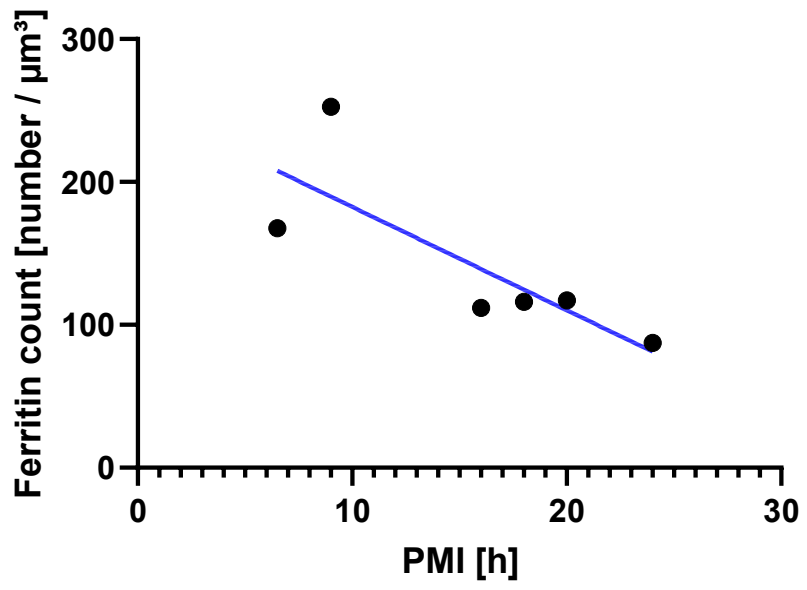

C
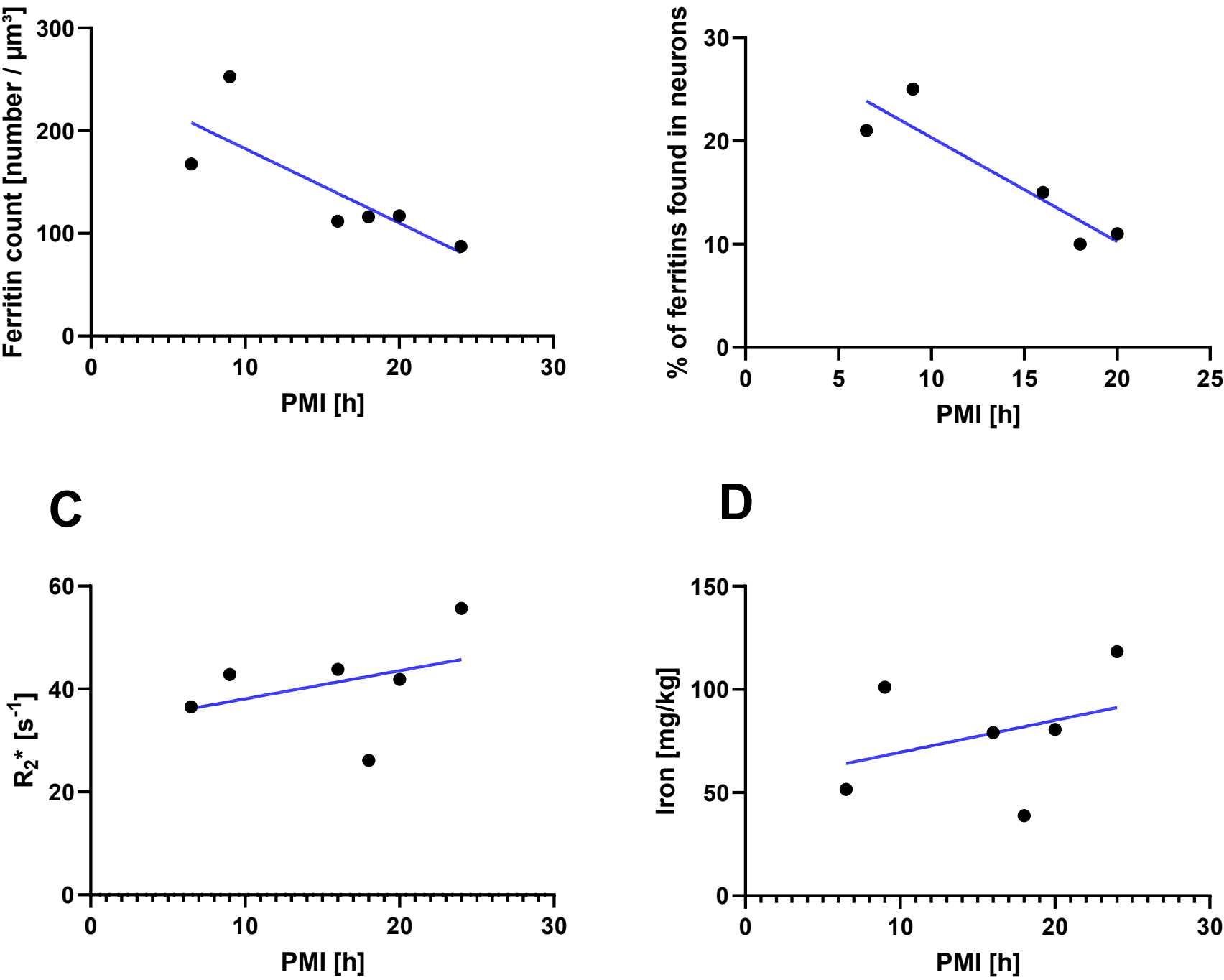